\title{
High-Sensitivity High-Stability Silicon Photodiodes for DUV, VUV and EUV Spectral Ranges"
}

\author{
L. Shi ${ }^{1}$, S. Nihtianov ${ }^{1}$, F. Scholze ${ }^{2}$, Gottwald, A. ${ }^{2}$, L. K. Nanver ${ }^{1}$ \\ ${ }^{1}$ Delft University of Technology, Mekelweg 4, 2628 CD Delft, The Netherlands \\ ${ }^{2}$ Physikalisch-Technische Bundesanstalt (PTB), Abbestraße 2-12, D-10587, Berlin, Germany
}

\begin{abstract}
In this paper, the optical and electrical performance of a newly developed silicon photodiode based on pure boron CVD technology (PureB-diodes) is introduced. Due to their extremely shallow p-n junction, with the depletion zone starting only a few nanometers below the surface, and nm-thin pure-boron-layer coverage of the anode surface, PureB-diodes have so far demonstrated the highest reported spectral responsivity in all sub-visible ultraviolet (UV) ranges: DUV (deep ultraviolet), VUV (vacuum ultraviolet) and EUV (extreme ultraviolet), covering a spectrum from $220 \mathrm{~nm}$ down to few nanometers [1][2][3][4]. Moreover, the measured responsivity at $13.5 \mathrm{~nm}$ wavelengths (EUV) approaches the theoretical maximum $(\sim 0.27 \mathrm{~A} / \mathrm{W})[5][6]$. PureB-diodes also maintain excellent electrical characteristics, with saturation-current values typical for high-quality silicon diodes, and a high breakdown voltage. Experimental results have demonstrated the extremely high radiation hardness of PureB-diodes when exposed to high EUV radiant exposures in the order of a few hundred $\mathrm{kJ} / \mathrm{cm}^{2}$ [4]. No change in the responsivity is observed within the experimental uncertainty. In the more challenging DUV and especially VUV ranges, PureB-diodes demonstrate a slight initial drop of responsivity (1 to $2 \%$ ), after which they stabilizes their performance [2].
\end{abstract}

Keywords: photodiode, ultrashallow junction, spectral responsivity, deep ultraviolet, vacuum ultraviolet, extreme ultraviolet.

\section{INTRODUCTION}

Recently, the demand for robust and high-performance photodetectors operating at wavelengths below $200 \mathrm{~nm}$, i.e. in the DUV (deep ultraviolet), VUV (vacuum ultraviolet) and EUV (extreme-ultraviolet) spectral ranges, has increased significantly, since applications profiting from these photodetectors can be found in space, in metrology and in lithography [7][8][9]. Therefore, the development and fabrication of high-performance detectors for these wavelength ranges are considered to be hot research topics. The availability of low-cost and well-developed silicon technology positions Si-based photodiodes as the main candidates for EUV radiation detection applications.

To guarantee a maximum internal quantum efficiency, and hence maximum spectral responsivity, pre-absorption of the incident radiation before the photodiode depletion zone has to be avoided. This is especially critical for VUV/DUV photons (100 nm - $300 \mathrm{~nm}$ wavelength), for which the attenuation length in silicon is smaller than 10 nanometers as shown in Figure 1 [10][11]. The best way to achieve this is to position the depletion zone as close to the device surface as possible [12]. Figure 2 presents the spectral responsivity of a Si-based ideal lossless system (no extra absorbing top layer) in the UV spectral range. The solid red line shows the upper limit given by the internal quantum efficiency of silicon. In the long-wavelength limit (red dashed line), one electron per absorbed photon is created and in the shortwavelength limit (red dotted line) a constant energy of $W \approx 3.66 \mathrm{eV}$ is needed to create one electron-hole pair, which leads to a constant responsivity $R=1 / W \approx 0.273 \mathrm{AW}^{-1}$. The solid blue line shows the spectral responsivity when the external reflection at the silicon surface is taken into account [5][6].

In this work the electrical and optical performance of PureB-photodiodes is presented. The experimental results show that these photodiodes demonstrate very high responsivity in the spectral range from $220 \mathrm{~nm}$ down to $1 \mathrm{~nm}$ wavelength. Moreover, the boron-doped $\mathrm{p}^{+} \mathrm{n}$ ultrashallow junction does not degrade during prolonged EUV/VUV exposure. In the past these diodes have been referred to as "B-layer" diodes [1]. The term "PureB" is introduced here to emphasis the fact that the amorphous boron layer that is deposited on the Si surface to form the anode, in itself plays an essential role in reducing the dark current [13] and also has the important function of preventing the formation of an insulating layer on the anode surface.

* This work is supported by The Dutch Technology Foundation STW, project number: 10024.

UV, X-Ray, and Gamma-Ray Space Instrumentation for Astronomy XVII, edited by Oswald H. Siegmund, Proc. of SPIE Vol. 8145, 81450N - (c) 2011 SPIE CCC code: $0277-786 X / 11 / \$ 18 \cdot$ doi: $10.1117 / 12.891865$ 


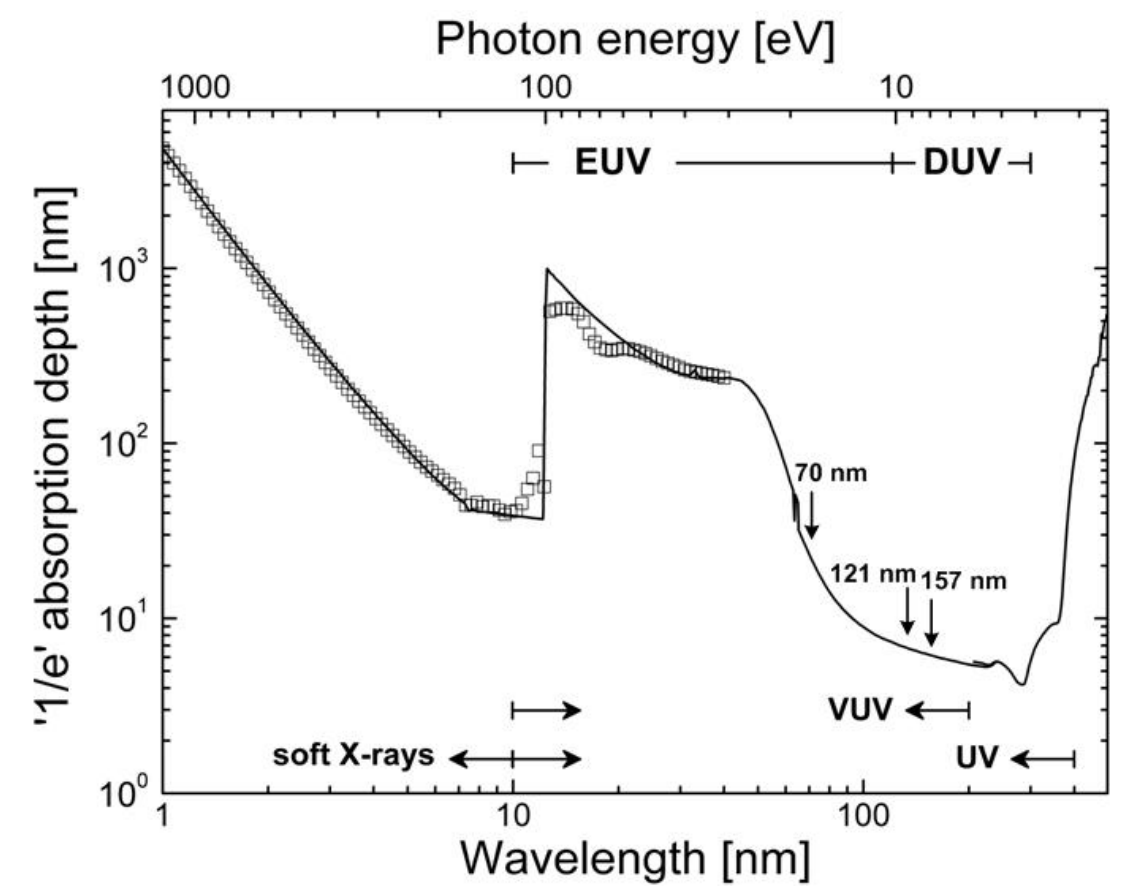

Figure 1. Penetration depth in Si vs. incident radiation wavelength / photon energy.

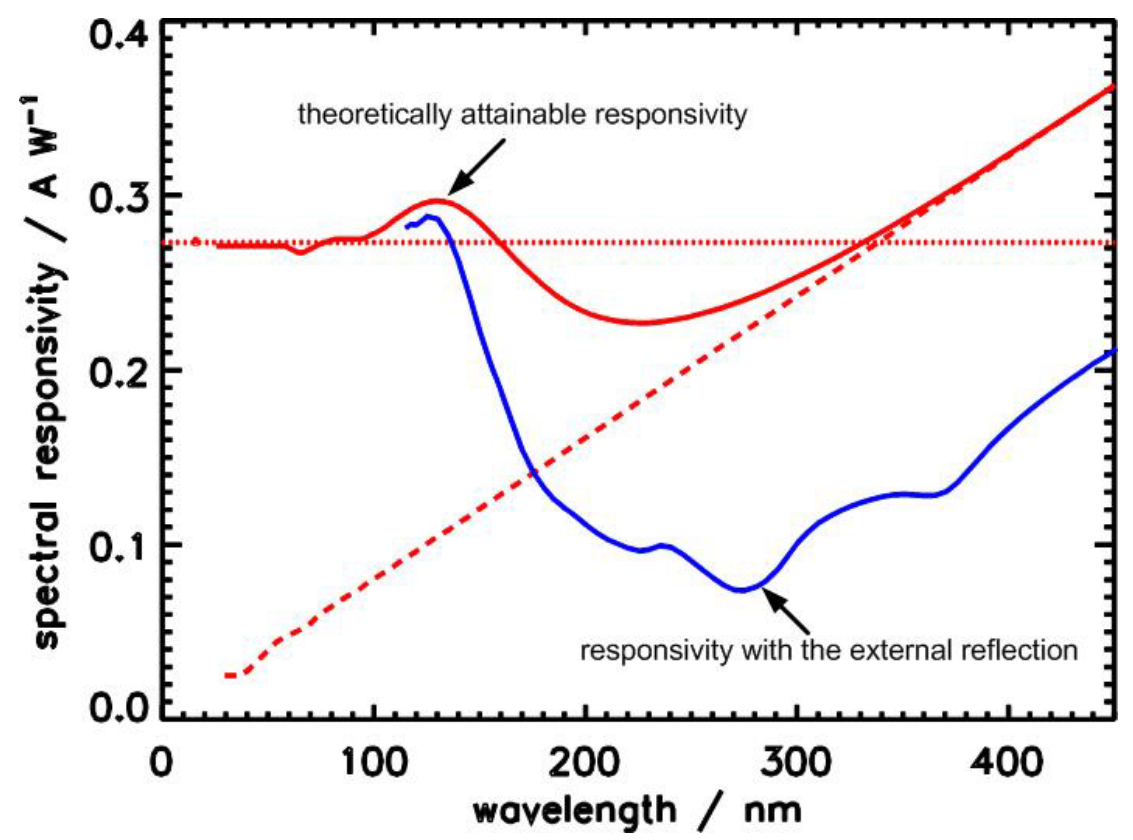

Figure 2. Ideal spectral responsivity of Si-based photodetectors.

\section{DEVICE DESCRIPTION}

The PureB-diode technology was developed at the DIMES (Delft Institute of Microsystems and Nanoelectronics) facilities of Delft University of Technology [3][13][14]. A schematic cross-section of the fabricated device is shown in Figure 3a. Figure $3 \mathrm{~b}$ compares a high-resolution TEM image of a PureB-layer formed after a 10 min deposition with the corresponding SIMS boron profiles [13][14]. 


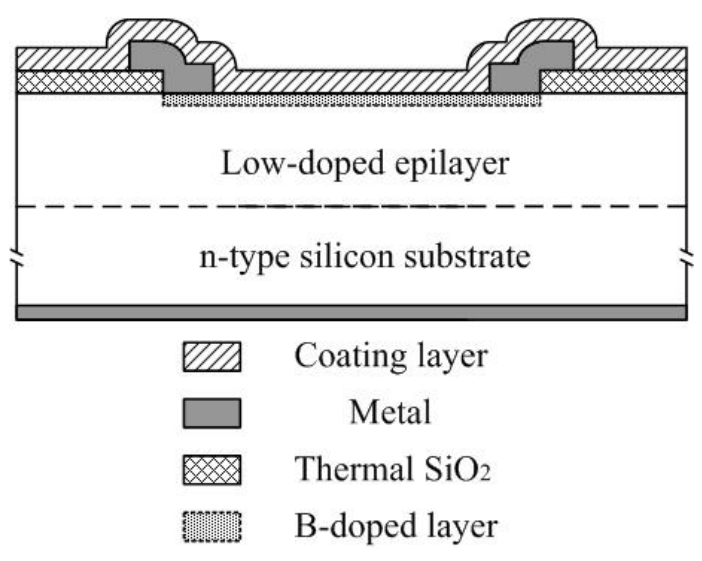

(a)

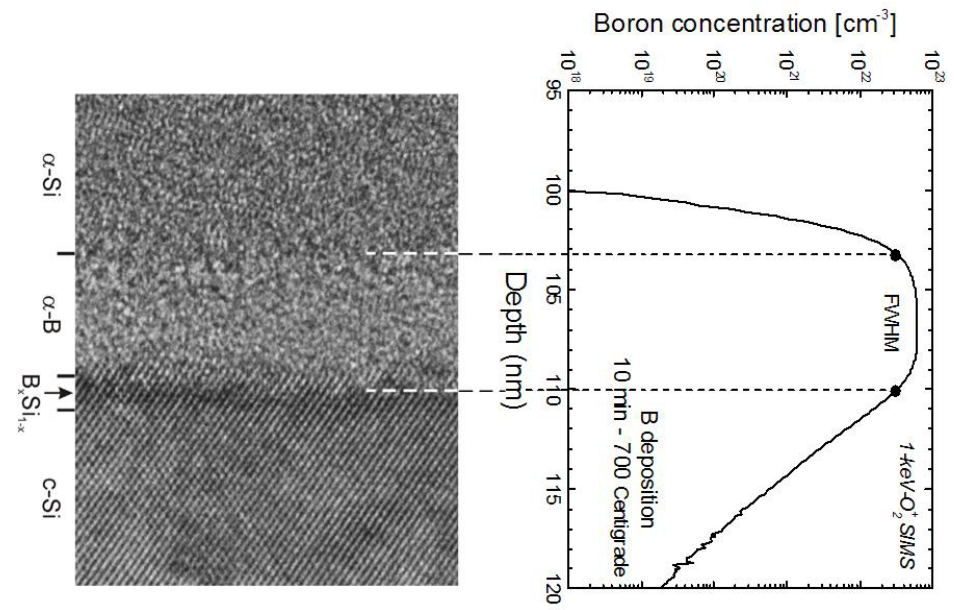

(b)

Figure 3. (a) Cross-section of PureB-layer diodes; (b) HRTEM image of a PureB-layer formed after a 10 min B deposition at $700{ }^{\circ} \mathrm{C}$ with a Boron SIMS profile. The sample was covered with $20 \mathrm{~nm}$ of PVD $\alpha$-Si for the TEM analysis.

A chemical vapour deposition (CVD) process of pure amorphous boron on n-type silicon is used, which results in a uniform nanometer-thin surface coverage of pure boron that gives an effective $\mathrm{p}^{+}$-doping of the Si surface thus forming a $\mathrm{p}^{+} \mathrm{n}$ junction [13][14]. Insulating or conductive coating layers can be optionally deposited directly onto this PureB layer, for example, to protect the surface or to facilitate the design and integration of thin-film filters with radiation spectrum pass-bands optimized for specific applications.

Typical I-V characteristics of PureB-diodes fabricated on a 4-inch, 2 - 5 ohm-cm, n-Si wafer are plotted in Figure 4a. The reproducibly low dark current and ideal I-V behavior indicate that the above-mentioned PureB-deposition process is a stable and reliable technology for making defect-free $p^{+} n$ junctions. Furthermore, as indicated in Figure $4 b$, the PureB-diodes also can maintain a very high breakdown voltage (an extremely low dark current is measured at a reverse bias of $-40 \mathrm{~V})[2]$.

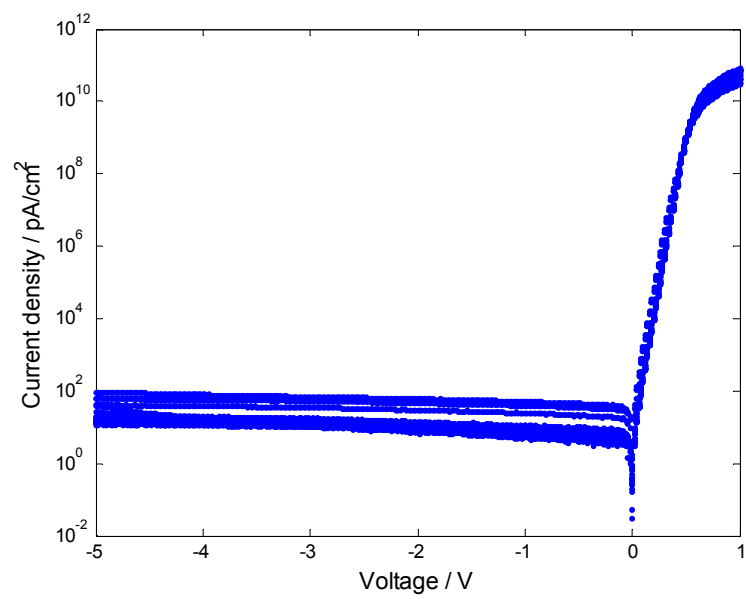

(a)

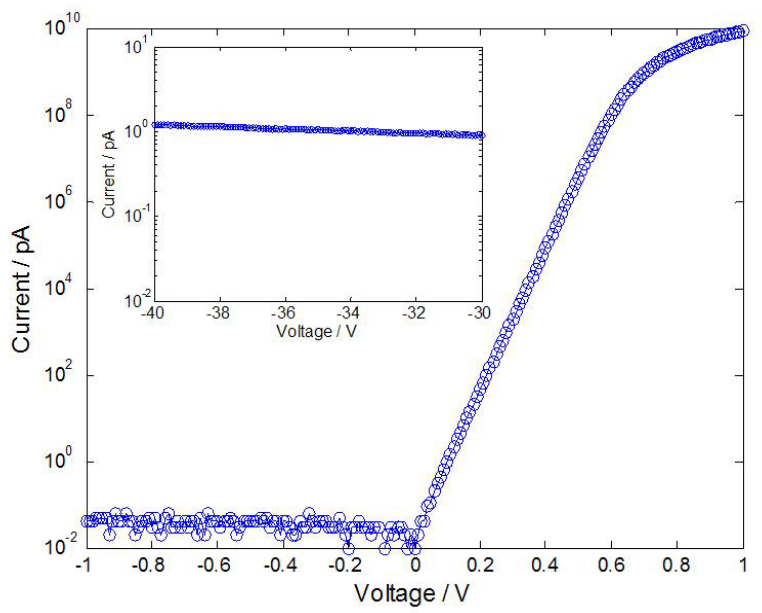

(b)

Figure 4. (a) I-V characteristics of 24 PureB-diodes (active area is $1 \times 1 \mathrm{~cm}^{2}$ ) fabricated on a 4-inch, $2-5 \mathrm{ohm}-\mathrm{cm}, \mathrm{n}-\mathrm{Si}$ wafer; (b) I-V characteristics of a B-layer diode (active area is $300 \times 300 \mu \mathrm{m}^{2}$ ) - the inset shows the dark current with a reverse bias up to $-40 \mathrm{~V}$. 


\section{OPTICAL PERFORMANCE}

All optical tests were carried out at the EUV- and UV/VUV-beamline synchrotron radiation laboratories of PTB (Physikalisch-Technische Bundesanstalt) in Berlin, Germany [15][16][17]. As shown in Figure 5a, an array of four photodiodes was assembled on a ceramic substrate. The positioning of the sample and the electrical contacts with the diodes were realized with a fixture mounted on an $x-y$ stage (indicated in Figure 5b) [4]. During each test, the detectors were in an ultra-high vacuum environment.

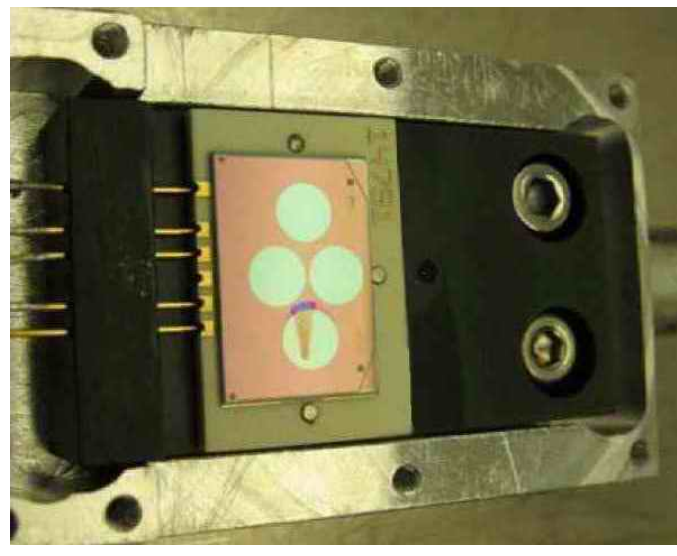

(a)

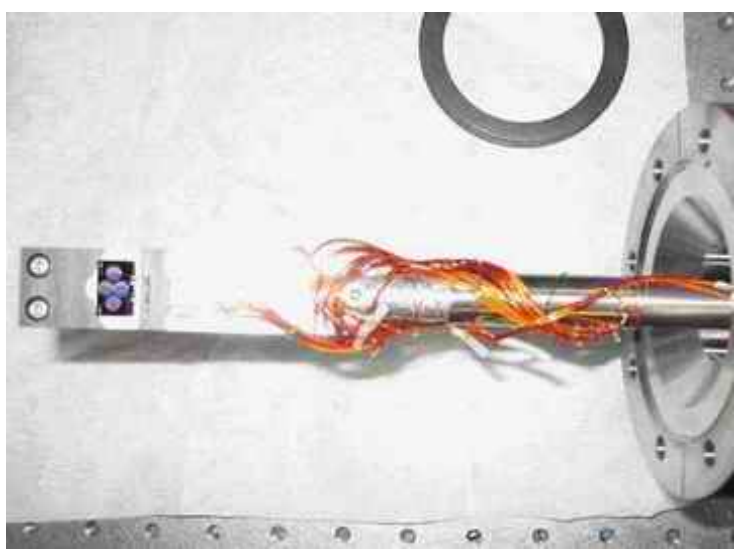

(b)

Figure 5. Mounting of the photodiode array on a PTB x-y positioning stage.

\subsection{EUV sensitivity}

The responsivity of a PureB-diode with a $\sim 3 \mathrm{~nm}$ amorphous boron top layer is shown in Figure 6. Clearly, the PureB diode performs with higher responsivity compared to commercially available state-of-the-art photodiodes [3][4]. At the $13.5 \mathrm{~nm}$ wavelength, the responsivity is about $0.27 \mathrm{~A} / \mathrm{W}$, which is essentially similar to that of an ideal lossless system [5][6]. This confirms that the B-deposition process can provide ultrashallow, high-quality, damage-free $p^{+}$-doped active surface layers which minimize any quantum-efficiency loss due to either recombination of photo-generated carriers or absorption in the front window.

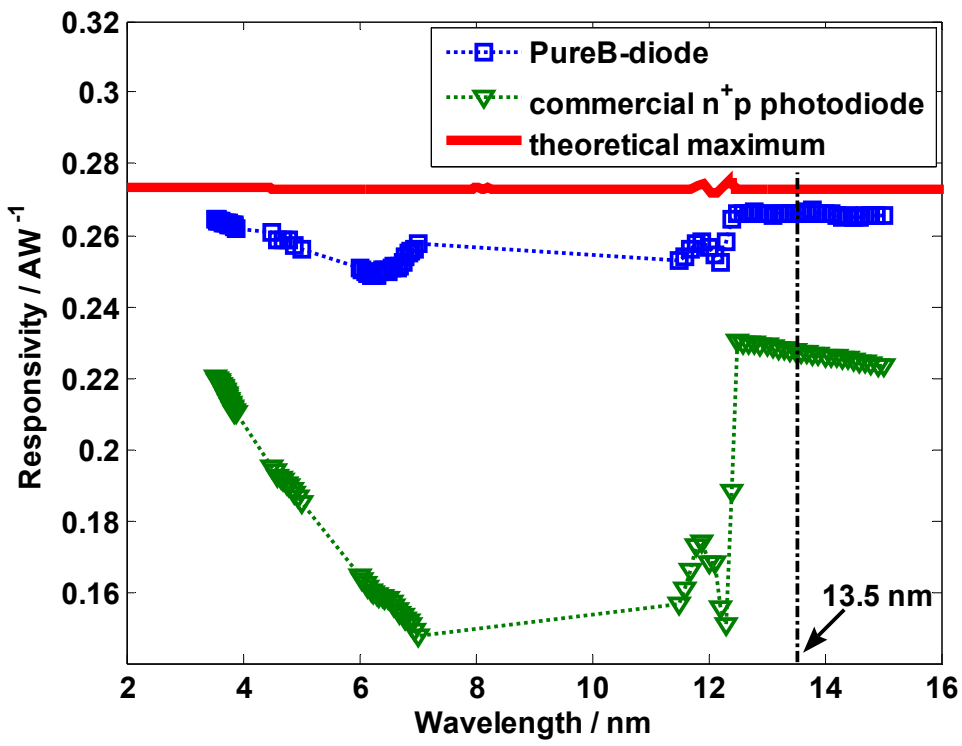

Figure 6. Measured spectral responsivity (symbols) of EUV PureB diodes with a 2.5 min B-deposition compared with a commercial $n^{+} p$ photodiode and the theoretically attainable values for an ideal Si-based photodetector. 


\subsection{DUV/VUV sensitivity}

As mentioned above, due to the extremely small penetration depth in $\mathrm{Si}$, DUV/VUV is the most challenging spectral range for Si-based photodetectors. Figure 7 shows the superior responsivity of the PureB-diode compared with other state-of-the-art photodiodes in the DUV/VUV spectral range (wavelengths from $50 \mathrm{~nm}$ to $215 \mathrm{~nm}$ ) [18][19][20]. The measured responsivity in the VUV range is in the order of $0.1 \mathrm{~A} / \mathrm{W}$ or higher [1][2].

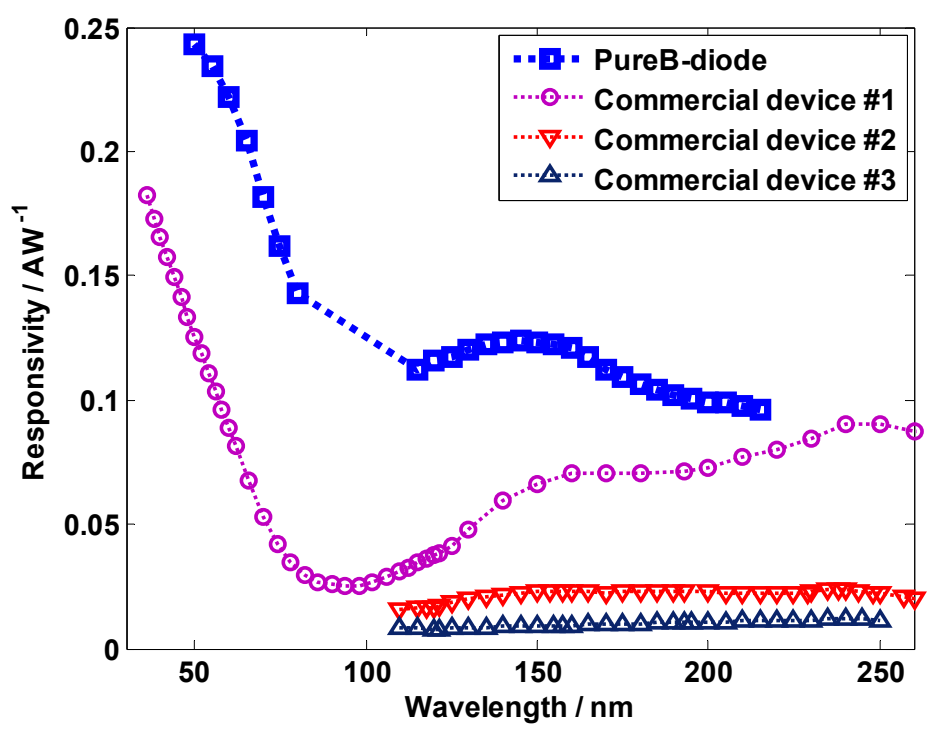

Figure 7. Measured responsivity of PureB-diodes in DUV/VUV spectral range compared with other state-of-the-art photodetectors.

\section{OPTICAL PERFORMANCE STABILITY}

Considering the nature of silicon (i.e. the relatively low strength of the chemical bonding structure), most of the conventional Si-based photodetectors demonstrate insufficient radiation hardness to high energy photons, especially in the EUV spectral range [21][22][23][24]. The responsivity degradation of the PureB-diode was evaluated by long-term exposures in the EUV and the VUV/DUV spectral ranges.

\subsection{EUV responsivity degradation}

The EUV radiation hardness of the PureB-diode was characterized by prolonged high radiant exposure $\left(0.22 \mathrm{MJ} / \mathrm{cm}^{2}\right.$ during $24.5 \mathrm{~h}$ ) to 13.5 -nm radiation [4][24]. The experimental result is presented in Figure 8a. The triangular symbols represent the ratio of responsivity after/before the high-intensity exposure. The observed degradation in spectral responsivity comes from a carbon contamination layer formed by the EUV radiation (Figure 8b) [25]. From the height of the carbon absorption edge in the spectrum around $4.3 \mathrm{~nm}$ as seen in Figure 8a, it can be calculated that the carbon layer has a thickness of about $20 \mathrm{~nm}$ [26]. The ratio of measured EUV spectral responsivity fits very well with the calculated ratio of responsivity of the same diode with/without a $20 \mathrm{~nm}$ carbon layer [27]. Therefore, it can be concluded that there is no measurable change in the responsivity due to degradation of the PureB-diode itself.

\subsection{VUV/DUV responsivity degradation}

For PureB-diodes with amorphous boron coverage of no more than $\sim 1 \mathrm{~nm}$ thick, a degradation of the responsivity was measured as indicated in Figure 9a. The measurements are performed immediately after a long term VUV exposure (107 $\mathrm{mJ} / \mathrm{cm}^{2}$ at $157 \mathrm{~nm}, 48 \mathrm{~mJ} / \mathrm{cm}^{2}$ at $121 \mathrm{~nm}$, and $28 \mathrm{~mJ} / \mathrm{cm}^{2}$ at $70 \mathrm{~nm}$ ). The responsivity recovers slowly with time due to a self-healing process [2]. The main cause for this degradation is believed to be the photo-electron-emission induced positive charging of the parasitic oxide top-layer (about a 10-nm-thick native oxide layer and $<1$-nm-thick boron layer on the diode surface were detected by ellipsometer). This is supported by the results of several linear scans to measure the responsivity across the detector surface at a wavelength of $193 \mathrm{~nm}$, as shown in Figure $9 \mathrm{~b}$. From the responsivity variation along the diode surface with time, the tendency of the irradiation-induced positive charge to spread and be discharged can be clearly distinguished. [2] 
It is believed that the oxide is formed due to oxidation of silicon through pinholes in the thin top boron layer when exposed to the environment. To prevent the formation of the nanometer-thin native oxide layer, which is charged during exposure, a longer boron deposition time is applied to form a thicker $(\sim 3 \mathrm{~nm})$ and more robust PureB-layer, as shown in Figure 10 [2]. Figure 11 indicates that PureB-diodes with a thicker boron layer demonstrate only a slight initial drop of responsivity (1 to $2 \%$ ), after which the responsivity stabilizes [1][2].

Although the photo-electron emission also occurs under EUV radiation, resulting in oxide charging to even higher voltage levels and hence a stronger electric field is created, the more than 500-nm penetration depth (shown in Figure 1) of EUV photons in silicon and boron makes the internal quantum efficiency basically insensitive to the deterioration of the surface charge collection.

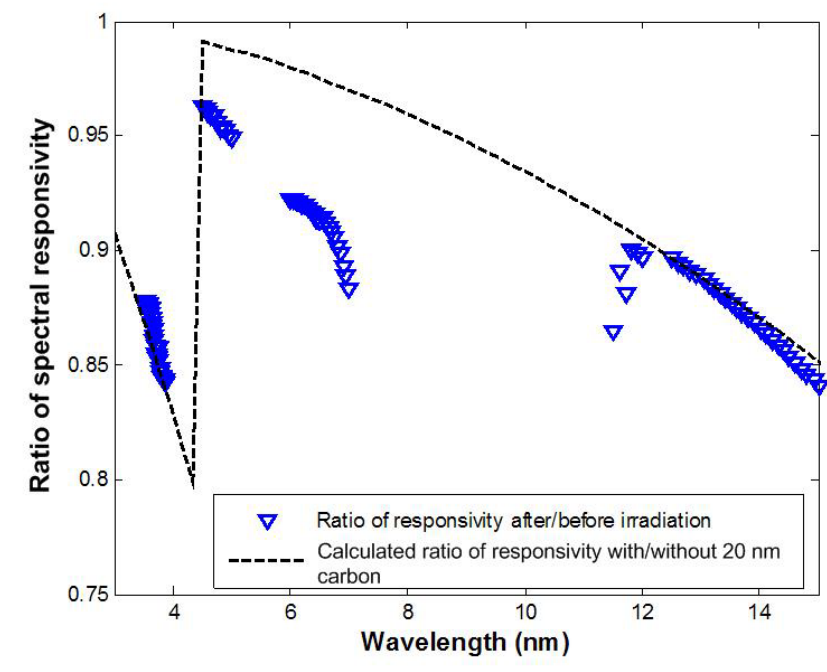

(a)
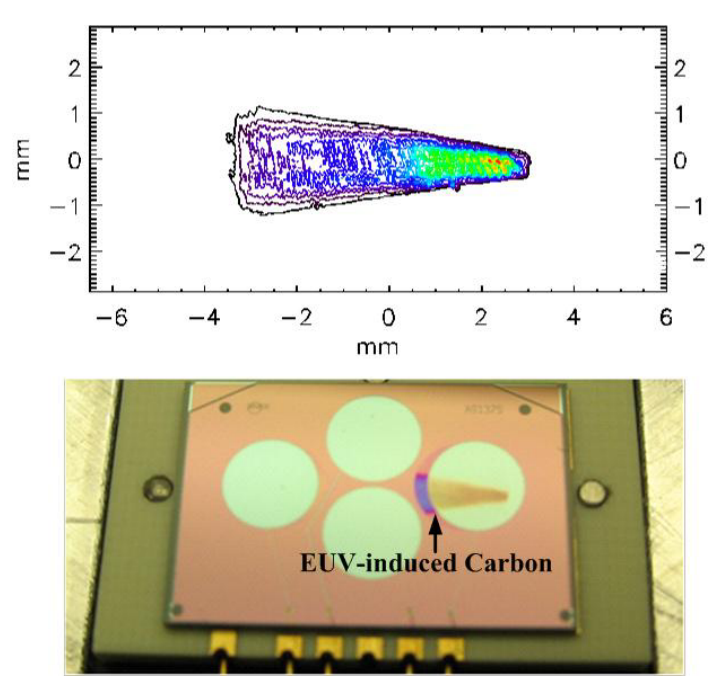

(b)

Figure 8. (a) Ratio of measured EUV spectral responsivity after/before intense EUV irradiation $\left(220 \mathrm{~kJ} / \mathrm{cm}^{2}\right)$, compared to the calculated ratio of responsivity based on the same diode with/without a $20 \mathrm{~nm}$ carbon layer; (b) shape of the EUV spot (irradiance in the high power region is $3 \mathrm{~W} / \mathrm{cm}^{2}[24]$ ) and the EUV-induced carbon contamination layer.

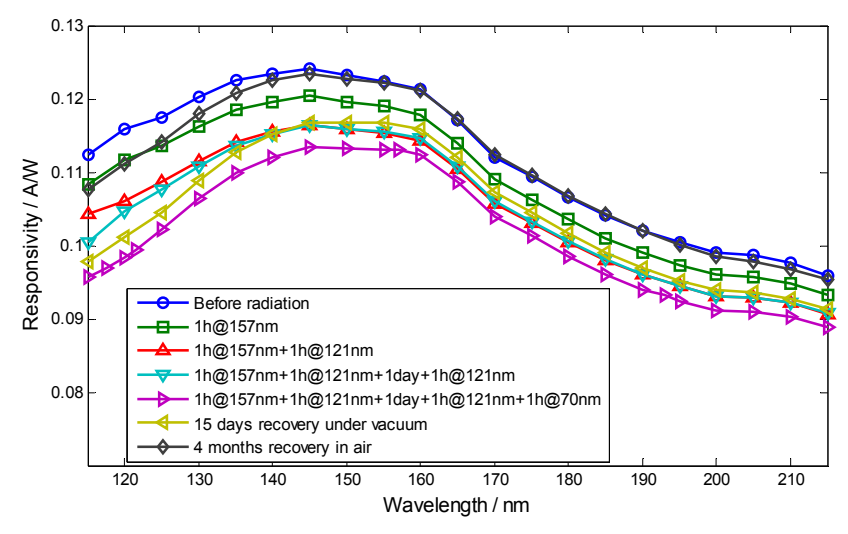

(a)

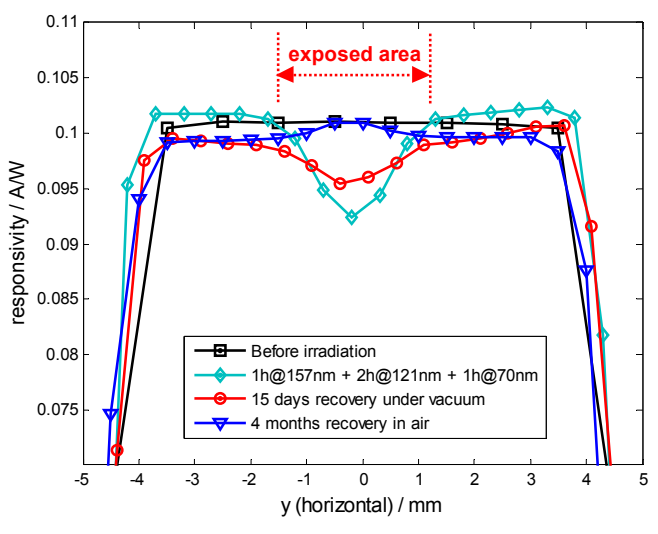

(b)

Figure 9. (a) Degradation of the VUV responsivity of a PureB-diode (with a $\sim 10$-nm native oxide layer and $<1$-nm boron layer on the diode surface) after a series of VUV/DUV irradiations; (b) measured responsivity variation along the diode surface over time of the same PureB-diode at a wavelength of 193-nm. VUV irradiations performed in the central region with radiant exposures as follows: $107 \mathrm{~mJ} / \mathrm{cm}^{2}$ at $157 \mathrm{~nm}, 48 \mathrm{~mJ} / \mathrm{cm}^{2}$ at $121 \mathrm{~nm}$, and $28 \mathrm{~mJ} / \mathrm{cm}^{2}$ at $70 \mathrm{~nm}$. 


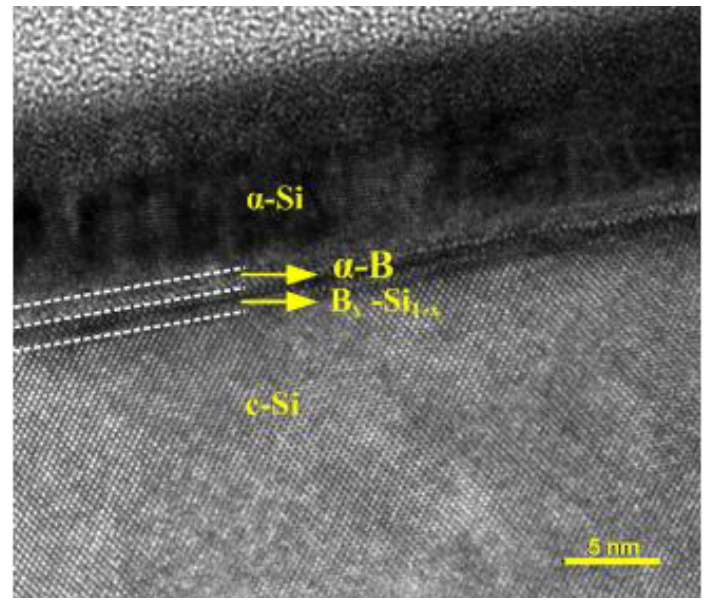

(a)

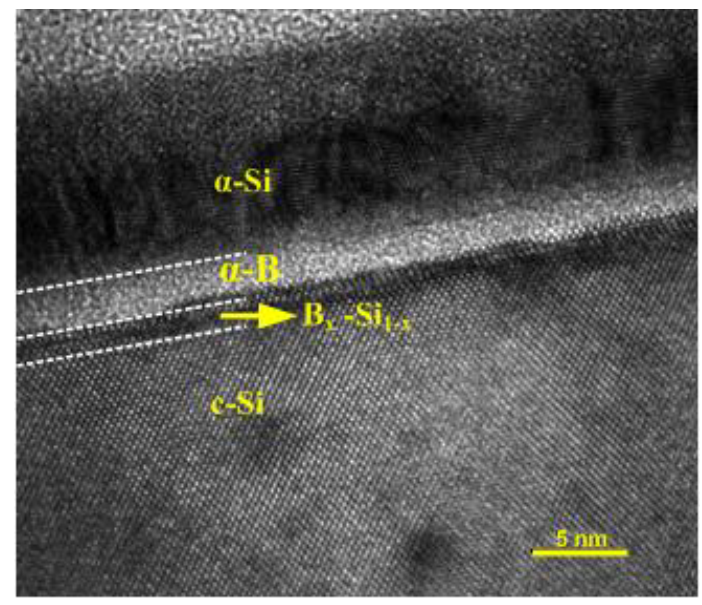

(b)

Figure 10. HRTEM images of (a) a 1-nm and (b) a 3-nm thick B-layer on a $\mathrm{Si}(100)$ substrate. The sample was covered with 20 nm PVD $\alpha$-Si for the TEM analysis.

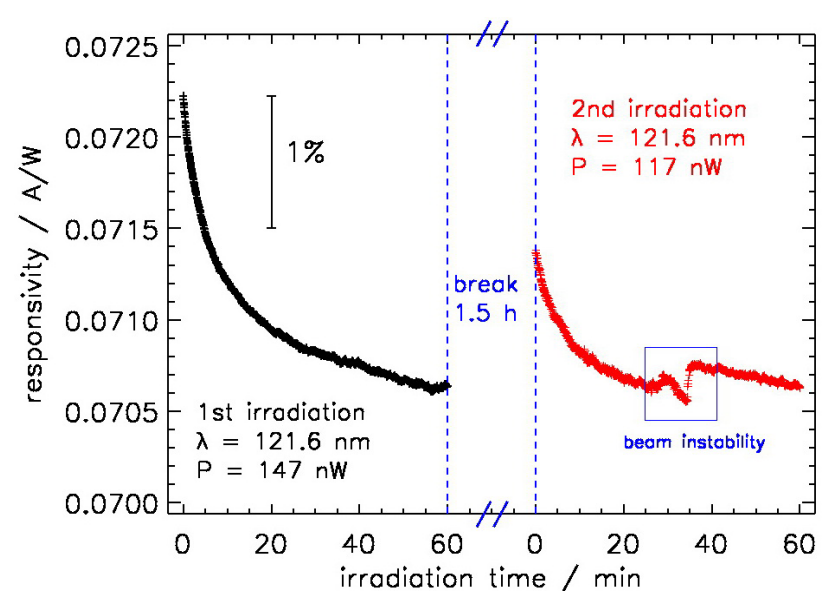

(a)

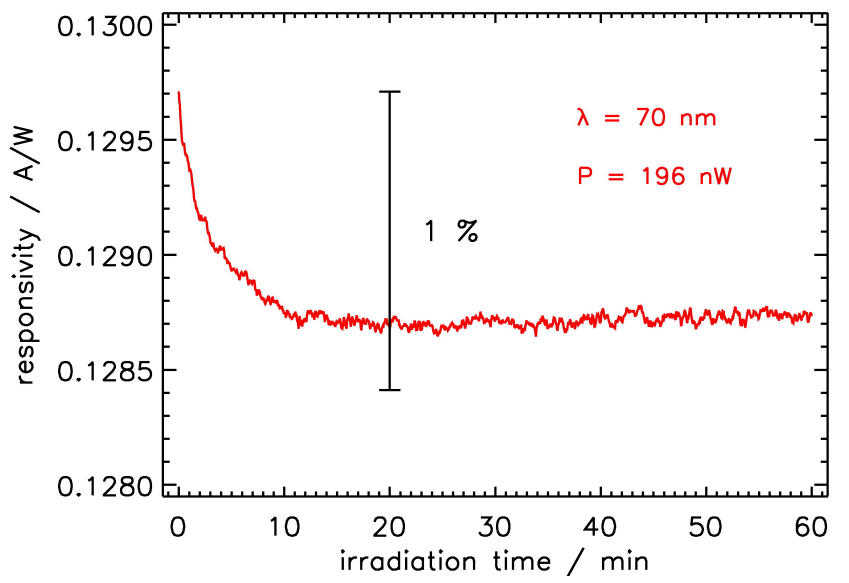

(b)

Figure 11. Degradation of the responsivity of PureB-diodes during irradiation (a) at $121.6 \mathrm{~nm}$ and (b) at $70 \mathrm{~nm}$.

\section{CONCLUSION}

In summary, we have presented a high-performance silicon-based photodiode for DUV, VUV and EUV radiation detection, covering a spectrum from $220 \mathrm{~nm}$ down to a few $\mathrm{nm}$ in wavelength. An outstanding electrical and optical performance has been achieved in terms of extremely low dark current, high responsivity and excellent stability to highdose VUV/EUV exposure. Such impressive features are achieved by a pure boron (PureB) CVD deposition technique for forming ultrashallow high-quality $\mathrm{p}^{+} \mathrm{n}$ junctions covered with an $\mathrm{nm}$-thin pure boron layer. A comparison with commercially available VUV/EUV detector solutions shows that the PureB-photodiodes perform with superior characteristics and exhibit a great potential for radiation detection in DUV, VUV and EUV based applications.

\section{ACKNOWLEDGMENT}

The authors would like to thank the staff of the DIMES-ICP cleanrooms and measurement room, in particular T.M.L. Scholtes and S. Milosavljević, for their support in the fabrication and measurement of the experimental material. 


\section{REFERENCES}

[1] Shi, L., Sarubbi, F., Nanver, L. K., Kroth, U., Gottwald, A., A., Nihtianov, S., "Optical performance of B-layer ultra-shallow-junction silicon photodiodes in the VUV spectral range", Proc. Eurosensors XXIV, Linz, Austria, September 5-8, (2010).

[2] Shi, L., Nanver, L. K., Šakić, A., Nihtianov, S., Gottwald, A.,Kroth,U., "Optical stability investigation of highperformance silicon-based VUV photodiodes", Proc. IEEESENSORS2010, Waikoloa, HI, USA, Nov. 1-4, pp. 132-135, (2010).

[3] Sarubbi, F., Nanver, L. K., Scholtes, T. L. M., Nihtianov, S., Scholze, F., "Pure boron-doped photodiodes: a solution for radiation detection in EUV lithography", Proc. IEEE 38th European Solid-State Device Research Conference (ESSDERC), Sept., pp. 278-281, (2008).

[4] Shi, L., Sarubbi, F., Nihtianov, S., Nanver, L. K., Scholtes, T. L. M., Scholze, F., "High performance siliconbased extreme ultraviolet (EUV) radiation detector for industrial application", Proc.IECON, Porto, Portugal, Nov. 3-5, pp. 1891-1896, (2009).

[5] Scholze, F., Rabus, H., Ulm, G., "Mean energy required to produce an electron-hole pair in silicon for photons of energies between 50 and 1500 eV", J. Appl. Phys., vol. 84, no. 5, pp. 2926-2939, Sept. (1998).

[6] Scholze, F., Henneken, H., Kuschnerus, P., Rabus, H., Richter, M., Ulm, G., "Determination of the electronhole pair creation energy for semiconductors from the spectral responsivity of photodiodes", Nuclear Instruments and Methods in Physics Research, A 439, pp. 208-215, (2000).

[7] Malinowski, P.E., et al., " $10 \mu \mathrm{m}$ pixel-to-pixel pitch hybrid backside illuminated AlGaN-on-Si imagers for solar blind EUV radiation detection", Proc. IEEE International Electron Devices Meeting (IEDM), San Francisco, CA, USA, Dec. 6-8, pp. 348-351, (2010).

[8] Blouke, M. M., Cowens, M. W., Hall, J. E., Westphal, J. A., Christensen, A. B., "Ultraviolet downconverting phosphor for use with silicon CCD imagers", Applied Optics, vol. 19, no. 19, pp. 3318-3321, (1980).

[9] International Technology Roadmap for Semiconductors: Lithography, Available at: http://www.itrs.net, Edition (2007)

[10] Palik, E. D., (Ed.), Handbook of Optical Constants of Solids, Orlando, FL: Academic Press, (1985).

[11] Henke data. Available from http://henke.lbl.gov/optical constants.

[12] US Patent 7586 108, Radiation detector, method for manufacturing a radiation detector and lithographic apparatus comprising a radiation detector, September 8, (2009).

[13] Sarubbi, F., Nanver, L. M., Scholtes, T. L. M., "High effective Gummel number of CVD boron layers in ultrashallow p ${ }^{+} \mathrm{n}$ diode configurations," IEEE Trans. Electron Devices, vol. 57, no. 6, pp. 1269-1278, (2010).

[14] Sarubbi, F., Nanver, L. K., Scholtes, T. L. M., "CVD delta-doped boron surface layers for ultra-shallow junction formation", ECS Transactions, vol. 3, no. 2, pp. 35-44, Nov. (2006).

[15] Gottwald, A., Kroth, U., Richter, M., Schöppe, H., Ulm, G., "UV and VUV detector-based radiometry at the MLS”, Meas. Sci. Technol. 21, 125101, (2010).

[16] Gottwald, A., Kroth, U., Krumrey, M., Richter, M., Scholze, F., Ulm, G., "The PTB high-accuracy spectral responsivity scale in the VUV and X-ray range", Metrologia, vol. 43, pp. S125-S129, (2006).

[17] Klein, R., Laubis, C., Müller, R., Scholze, F., Ulm, G., "The EUV metrology program of PTB", Microelectronic Engineering, vol. 83, pp. 707-709, (2006).

[18] Richter, M., Kroth, U., Gottwald, A., Gerth, C., Tiedtke, K., Saito, T., Tassy, I., Vogler, K., "Metrology of pulsed radiation for 157-nm lithography", Appl Opt., vol. 41, no. 34, pp. 7167-7172, Dec. (2002).

[19] Canfield, L. R., Vest, R. E., Korde, R., Schmidtke, H., Desor, R., "Absolute silicon photodiodes for $160 \mathrm{~nm}$ to $254 \mathrm{~nm}$ photons", Metrologia, vol. 35, p. 329, (1998).

[20] Solt, K., Melchior, H., Kroth, U., Kuschnerus, P., Persch, V., Rabus, H., Richter, M., Ulm, G., "PtSi-n-Si Schottky-barrier photodetectors with stable spectral responsivity in the 120-250 nm spectral range", Appl. Phys. Lett., vol. 69, no. 24, pp. 3662-3664, Dec. (1996).

[21] Scholze, F., Klein, R., Müller, R., "Characterization of detectors for extreme UV radiation", Metrologia, vol. 43, pp. S6-S10, (2006).

[22] Scholze, F., Brandt, G., Müller, P., Meyer, B., Scholz, F., Tümmler, J., Vogel, K., Ulm, G., "High-accuracy detector calibration for EUV metrology at PTB", Proc. SPIE 4688, pp. 680-689, (2002).

[23] Vest, R. E., Canfield, L. R., "Evaluation of $\mathrm{Au} / \mathrm{GaAsP}$ and $\mathrm{Au} / \mathrm{GaP}$ Schottky photodiodes as radiometric detectors in the EUV", Rev. Sci. Instrum., vol. 67, p. 3362, (1996). 
[24] Klein, R., Gottwald, A., Scholze, F., Thornagel, R., Tümmler, J., Ulm, G., Wedowski, M., Stietz, F., Mertens, B., Koster, N., Elp, J. V., "Lifetime testing of EUV optics using intense synchrotron radiation at the PTB radiometry laboratory", Soft X-Ray and EUV Imaging Systems II, D. A. Tichenor and J. A.Folta, eds., Proc. SPIE 4506, pp. 105-112, (2001).

[25]Garg, R., Wüest, A., Gullikson, E., Bajt, S., Denbeaux, G., "EUV optics contamination studies in presence of selected hydrocarbons", Proc. of SPIE., vol. 6921, p. 692136, (2008).

[26] Calculation performed with NIST software at: http://physics.nist.gov/PhysRefData/FFast/html/form.html.

[27] Filter Transmission database. Available at: http://henke.lbl.gov/optical_constants/filter2.html. 